\title{
Análisis de la Conversación: Psicoterapia de adolescentes depresivos con intento de suicidio en contexto hospitalario
}

\section{Analysis of the Conversation: Psychotherapy of depressed adolescents with attempted suicide in a hospital context}

\author{
Lautaro Barriga \\ Universidad Bernardo O Higgins. Escuela de Psicología. Santiago de Chile \\ Universidad Gabriel Mistral. Escuela de Psicología. Santiago de Chile. \\ Marco Villalta \\ Universidad de Santiago de Chile. Escuela de Psicología. Santiago de Chile
}

Recibido (26 de junio de 2018) Aceptado (28 de noviembre de 2018)

\begin{abstract}
Resumen
Investigaciones recientes en psicoterapia muestran que el estudio de la interacción psicoterapéutica al centrar su atención en la conversación de interlocutores, entrega señales que permiten analizar el cambio terapéutico. El propósito del presente estudio de caso es analizar el movimiento de una Estructura de Intercambios Terapéuticos en la conversación de procesos psicoterapéuticos completos de adolescentes depresivos con intento de suicidio en contexto hospitalario. Se analizó el uso del tiempo de los intercambios comunicacionales entre terapeuta y paciente durante las sesiones, que fueron segmentadas en inicio, desarrollo y cierre. Los resultados muestran que los intercambios se producen según su utilidad en la psicoterapia y que su ocupación es sensible y ajustada al trabajo y objetivos terapéuticos que van surgiendo en la interacción. Adicionalmente se identificaron intercambios que son funcionales al inicio, desarrollo y cierre de cada sesión, y otros que deben utilizarse con cautela por parte del psicoterapeuta. La investigación verifico que el psicoterapeuta debe saber cuál, cuándo y cómo utilizar la Estructura de Intercambios Terapéuticos.

Palabras clave: Intercambios; Cambio en Psicoterapia; Adolescentes; Análisis de la Conversación.
\end{abstract}

\begin{abstract}
Recent research in psychotherapy shows that the study of psychotherapeutic interaction by focusing attention on the conversation of interlocutors, provides signals to analyze the therapeutic change. The purpose of the present case study is to understand the movement of a Therapeutic Exchange Structure in the conversation of complete psychotherapeutic processes of depressed adolescents with attempted suicide in a hospital context, analyzing the use of the time of the exchanges in the sessions, which were segmented in start, development and closure. The results show that the exchanges are managed according to their usefulness in psychotherapy that their occupation is sensitive and adjusted to the work and therapeutic objectives that arise in the interaction. In addition, there would be exchanges that are functional at the beginning, development and closure of each session, and others that should be used with caution. The psychotherapist must know what, when and how to use the Structure of Therapeutic Exchanges.

Key words: Exchanges; Change in Psychotherapy; Teenagers; Analysis of the Conversation.
\end{abstract}

Correspondencia: Lautaro Barriga Carvajal, E-Mail: lautaro.barriga@ubo.cl General Gana N 1702. Santiago de Chile. Estudio auspiciado por el Fondo Nacional de Desarrollo Científico y Tecnológico (FONDECYT) ํo 1150237 


\section{Introducción}

La adolescencia es una etapa de cambios significativos donde se configura la identidad y la personalidad, sin embargo, antes situaciones de estrés, rupturas con personas significativas, pérdida de seres queridos, problemas relacionales, acontecimientos de humillación, degradación o vergüenza, pueden alterar el desarrollo psicológico y activar conductas de riesgo como pensamientos y actos suicidas (Barrientos \& Cárdenas, 2013; Ministerio de Salud de Chile [MINSAL], 2013).

El suicidio de adolescentes en Chile constituye la segunda causa de muerte entre los 10 a 24 años de edad en población general, y la tercera causa de muerte en hombres adolescentes (Guajardo, Ojeda, Achui \& Larraguibel, 2015). La prevalencia de ideación suicida alcanza un $62 \%$ en adolescentes, es decir, de cada tres jóvenes de enseñanza secundaria que piensan que la vida no tiene sentido, dos de ellos planifican su suicidio y uno lo intenta (Vicente, Saldivia, Rioseco, De la Barra, Valdivia, Melipillan, Zúñiga, Escobar \& Pihán, 2010; Vicente, Saldivia \& Pihán, 2016). Chile es el país Latinoamericano que más incrementó sus tasas de suicidio adolescente, por esto el MINSAL lo considera como un problema de salud pública y formula un objetivo sanitario en su Estrategia Nacional de Salud para la década del 2011-2020, cuyo fin es reducir la tasa de suicidio en este grupo etario (MINSAL, 2013). Ahora bien, adolescentes con ideación e intentos de suicidio, según su gravedad, necesitan estabilización médica y psicoterapia (Almonte \& Montt, 2012).

Los adolescentes no siempre presentan motivación para el tratamiento, tienen dificultad para establecer la alianza terapéutica y no existe una clara conciencia del problema, en consecuencia, la necesidad de ayuda y de requerirla no es una condición como en el adulto (Almonte \& Montt, 2012). En este sentido, obtener conocimiento específico de las diversas variables que inciden en la psicoterapia de adolescentes es fundamental para la eficacia terapéutica. En la actualidad, el foco de atención y de exploración esta puesto en el proceso, cobrando gran relevancia en la psicoterapia los estudios del lenguaje en la interacción de cambio terapéutico (Aristegui, Reyes, Tomicic, Vilches, Krause, De la Parra, Ben Dov, Dagnino, Echávarri, \& Valdés, 2004).

Conocer el dialogo psicoterapéutico de adolescentes depresivos con intento de suicidio grave, permite comprender cómo se van construyendo los significados y el cambio en un determinado contexto, como el hospitalario, lugar donde los procedimientos de atención se rigen por políticas públicas.

Una perspectiva de análisis que explora y permite comprender la construcción interactiva de los diálogos en la psicoterapia es el Análisis de la Conversación (AC), que se focaliza en la inter-relación detallada de procesos se- cuenciales e intersubjetivos producidos en la interdependencia de interlocutores situados en contextos específicos (Peräkyla, 2004; Villalta, 2009). En este sentido, el lenguaje desde su función interactiva - ilocutiva y perlocutiva - integra los niveles jerárquicos de la lingüística interaccional con la etnografía de la comunicación (Kebrat-Orecchioni, 1998; Hernández, 2000; Villalta, 2009), dando cuenta del concepto de Intercambio - unidad mínima del lenguaje en la mirada dialogal — que permite describir a la psicoterapia como una estructura dialogal co-construida, colaborativa y vinculante de interlocutores ubicados en un determinado contexto.

Observar los diálogos psicoterapéuticos organizados en intercambios permite, desde la evidencia, levantar conocimiento de tratamientos efectivos de adolescentes. En este sentido, el presente estudio se focaliza en comprender los movimientos de una Estructura de Intercambios Terapéuticos durante la conversación psicoterapéutica de procesos completos de adolescentes depresivos con intento de suicidio en contexto de salud pública hospitalaria, analizando las características del uso de los tiempos de los intercambios producidos en las sesiones, las que fueron segmentadas en inicio, desarrollo y cierre.

\section{Suicidio adolescente: Problema de salud pública}

El suicidio adolescente presenta múltiples factores de riesgo, entre ellos el trastorno depresivo, siendo el cuadro clínico más relevante y con mayor carga de morbilidad entre los jóvenes (MINSAL, 2013). Las estadísticas ubican a Chile en el segundo país en el mundo y el primero de Latinoamérica con mayores tasas de suicidio (Organización para la Cooperación y el Desarrollo Económico [OCDE], 2013). El suicidio de adolescentes en Chile fue de 5,83 por 100.000 habitantes el año 1990 , de 9,28 por 100.000 habitantes el año 2005 y de 11,8 por 100.000 el año 2011, triplicándose entre los 10 y 29 años de edad (Guajardo et al., 2015). El suicidio es la segunda causa de muerte entre los 10 a 24 años de edad en población general y la ideación suicida se presenta en un $62 \%$ de los adolescentes, es decir, de cada tres jóvenes que cursa enseñanza media y creen que la vida vale nada, dos de ellos piensan en suicidarse y uno lo intenta, reflejando la fuerza del fenómeno en este grupo etario que en la actualidad se incrementa sostenidamente entre los 10 a 19 años de edad, llegando a 12 casos de suicidio por cada 100.000 habitantes (Vicente et al., 2010; Vicente, Saldivia \& Pihánl, 2016; MINSAL, 2013).

Las mujeres adolescentes presentan mayor frecuencia de intentos de suicidio, pero son los hombres quienes lo consuman. El suicidio de hombres adolescentes presenta una proporción de tres a cinco veces más que las mujeres, siendo la tercera causa de muerte de hombres adolescentes, luego de accidentes y homicidios (Guajardo et al., 2015). 
A pesar que los hombres adolescentes se suicidan más que las mujeres, el suicidio es la causa más común de muerte en las mujeres adolescentes entre los 15 a 19 años de edad (Jiménez y Aceituno, 2013). Las estadísticas de suicidio infanto-juvenil impactan negativamente a la sociedad, ya que el suicidio de un niño o adolescente genera marcada perturbación a nivel familiar, escolar y social, encendiendo alarmas en el MINSAL, institución que lo considera como problema de salud pública y lo incorpora en su Estrategia Nacional de Salud período 2011-2020 como un objetivo sanitario que busca reducir el suicidio adolescente (MINSAL, 2013). El énfasis del MINSAL está puesto en la reflexión, asistencia sanitaria, abordaje terapéutico y fortalecimiento de los servicios de salud mental de la red pública y privada en sus distintos niveles de atención - primario, secundario, terciario-, que de acuerdo a la gravedad del intento de suicidio - leve, moderado y grave-, el sistema de salud terciario u hospitalario es el dispositivo que responde a los casos de mayor gravedad (MINSAL, 2013).

Servicios de salud mental de adolescentes en contexto hospitalario ofertan prestaciones ambulatorias y de hospitalización. En ambas prestaciones, la indicación central a trabajar con los intentos de suicidio es la psicoterapia (Almonte \& Montt, 2012; Guajardo et al., 2015).

\section{Psicoterapia de adolescente en contexto hospitalario}

Los hospitales son definidos por el MINSAL como establecimientos de alta complejidad técnica, responsables de proveer atenciones médicas, cuidados intensivos e intermedios a la población beneficiaria y desarrollar actividades de promoción, prevención, curación, rehabilitación y cuidados paliativos (MINSAL, 2000; MINSAL, 2013). En este sentido, el contexto hospitalario otorga atención sanitaria a patologías de alta complejidad, porque presentan mayores riesgos asociados y necesitan de procedimientos, tratamientos y tratantes altamente especializados, regulado por protocolos con altos estándares de calidad. Un dispositivo de alta complejidad adquiere la condición de centro de referencia nacional, es decir, un organismo sanitario que recibe a usuarios derivados de diferentes zonas del país.

En general, los psicólogos que trabajan en los hospitales chilenos son psicólogos clínicos especialistas en psicoterapia, acreditados por la comisión nacional de acreditación de psicólogos clínicos de Chile (CONAPC), es decir, adquirir la condición de psicoterapeutas implica recibir una formación de post-título para cumplir con los estándares del cargo (Sallés, 2011). Los post-título tienen distintos enfoques de psicoterapia, en consecuencia, se espera que en el contexto hospitalario convivan diferentes modelos psicoterapéuticos trabajando con las mismas condiciones de espacio, tiempo, procedimientos y protocolos de atención. Por lo tanto, los psicoterapeutas de hospitales que atienden a adolescentes responden a la demanda de atención adaptándose a lo establecido por la institución hospitalaria.

Se ha documentado que la psicoterapia de adolescentes necesita mayor investigación, ya que descripciones de la alianza terapéutica, el clima terapéutico y auto-conocimiento del psicoterapeuta, se basan principalmente en estudios $\mathrm{y}$ en adaptaciones de intervenciones que han funcionado para los adultos (Baylis, Collins \& Coleman, 2011; Vargas, Gumucio \& González, 2015). Por lo tanto, es imprescindible proporcionar, desde la evidencia, estrategias psicoterapéuticas eficaces para este grupo etario, ya que de cómo se resuelvan los conflictos en este periodo de desarrollo, será la madurez y estabilidad del adulto (Forman, 2004). Lo anterior implica la necesidad de ahondar en el proceso interactivo de la psicoterapia de adolescentes, siendo una alternativa para observar y comprender esta interacción el modelo de análisis de la conversación.

\section{Análisis de la Conversación (AC): La interacción psicoterapéutica del adolescente.}

El AC es un modelo de análisis desarrollado por Sacks, Schegloff \& Jefferson (1974), quienes observan que los interlocutores presentan procedimientos compartidos al comunicarse y métodos para entender lo que el otro está intentando decir o hacer desde lo que está hablando. Esto es posible porque cada emisión verbal se vincula cooperativamente con la otra, configurando una secuencia mutuamente comprensiva dentro de un segmento del dialogo que estos autores llamaron "estructura de interacción del par adyacente u organización secuencial básica de la interacción" (Sacks, Schegloff \& Jefferson, 1974).

Los estudios de interacción terapéutica miran en detalle lo que sucede momento a momento en cada sesión de psicoterapia (Gaete, Aristegui \& Krause, 2017). Barriga (2017) plantea que el AC hace posible que en las sesiones se reconozca un tipo de conversación especializada, que se organiza colaborativamente en secuencias de diálogos con reglas y normas que determinan los propósitos y las acciones interdependientes entre los interlocutores durante la conversación. Esta noción cooperativa de la conversación permite que el lenguaje privado del adolescente se conozca y se organice en la psicoterapia, definiendo y objetivando su problemática. En este sentido, el lenguaje en la conversación psicoterapéutica es una forma de interacción que compromete a los interlocutores en su construcción, creando un campo de acción organizado que mantiene las intersubjetividades (Peräkyla, 2004; Villalta, 2009). En consecuencia, la interacción psicoterapéutica es una co-construcción con normas, procedimientos y de acción conjunta e interdependiente entre psicoterapeuta-consultante, que mantiene las intersubjetividades de ambos interlocutores, los confirma 
en sus identidades y los orienta al logro de sus propósitos y de sus objetivos en un contexto psicoterapéutico particular, siempre vinculados por el lenguaje en su función interactiva —ilocutiva y perlocutiva— (Peräkyla, 2004; Pomerantz \& Fehr 2000; Kondratyuk \& Peräkyla, 2011).

La interacción psicoterapéutica ha sido abordada en perspectiva del $\mathrm{AC}$ en niveles jerárquicos de análisis y en contexto para dar cuenta de las estructuras dialogales vinculadas a distintos procesos de psicoterapia en población adolescente (Barriga, 2017). Un primer nivel de análisis sitúa a las fases o dimensión temporal, que describe una organización que marca un inicio, un desarrollo y un cierre en cada sesión. $\mathrm{Al}$ interior de cada fase se desarrollan bloques o secuencias temáticas, que son temas de conversación conectados, secuenciados y caracterizados por una fuerte coherencia pragmática y semántica. Dentro de estos bloques o secuencias se encuentran el Intercambio, que es la unidad mínima de la conversación desde una perspectiva dialogal, compuesta al menos de dos intervenciones y dos interlocutores distintos, vinculados por la acción cooperativa para obtener significados, buscar la satisfacción interaccional y lograr acuerdos compartidos sobre la intervención inicial de un interlocutor (Kebrat-Orecchioni, 1998; Villalta, 2009; Barriga, 2017). Los Intercambios son delimitados temporalmente y su análisis hace posible reconocer una organización de diálogos como una estructura conversacional intersubjetiva y etnográfica que se arma durante la interacción en un determinado contexto (Villalta, 2009; Barriga, 2017). Los intercambios se encuentran conformados por intervenciones o acciones comunicativas orientadas mutuamente en la interacción. La intervención en investigación ha sido de tipo verbal y de interpretación del comportamiento no verbal de los interlocutores situados en contexto.

Se encontró en procesos psicoterapéuticos de adolescentes en contexto hospitalario una conversación organizada en fases, secuencias, tipos de intervenciones y "Estructura de Intercambios Terapéuticos" (Barriga, 2017).

\section{Método}

\section{Diseño de Investigación}

Método cualitativo de tipo exploratorio y descriptivo en la línea de la lingüística interaccional, que combina técnicas cualitativas de observación etnográficas de análisis de la conversación con herramientas cuantitativas de medición, asociados a datos de registros de observación categorizados del proceso de psicoterapia.

\section{Participantes}

Estudio de casos múltiples, usados frecuentemente en investigaciones clínicas de medicina, psicología y psicotera- pia (Stake, 2005).

Los casos de estudio son dos procesos psicoterapéuticos completos obtenidos de un servicio de psiquiatría y salud mental infanto-juvenil de un hospital público de la cuidad de Santiago de Chile. Se eligió este servicio por sus prestaciones de hospitalización, por contar con protocolos acreditados por la autoridad sanitaria y porque los psicólogos que trabajan en la institución se encuentran acreditados como especialistas en psicoterapia por la CONAPC. Esto último implica que en contexto hospitalario, los distintos modelos psicoterapéuticos actúan bajo los mismos procedimientos que dicta la institución. Los casos de estudio contienen 14 sesiones cada uno, de las cuales se filmaron y analizaron las sesiones 1. ${ }^{\mathrm{a}}, 3 .^{\mathrm{a}}, 6 .^{\mathrm{a}}, 9 .^{\mathrm{a}}, 12 .^{\mathrm{a}}$ y $14 .^{\mathrm{a}}$, es ecir, la sesión que abre, cuatro sesiones del movimiento terapéutico y la sesión que cierra. Los criterios de inclusión de los participantes fueron:

- Psicoterapeuta psicólogo: Que trabaje en el servicio de psiquiatría y salud mental infanto-juvenil, permanencia laboral mínimo de 5 años, formación psicoterapéutica certificada por la CONAPC y experiencia en atención adolescente mínima de 5 años.

- Consultante adolescente: Mujeres entre 14 y 16 años de edad, cursando educación secundaria en jornada diurna, derivada de la red pública de salud, con diagnóstico de depresión e intento de suicidio grave por intoxicación con fármacos u otros compuestos químicos, con adulto responsable que autorice la hospitalización en salud mental e intervenciones previas en centros de salud mental en los últimos seis meses. Se excluyó psicosis, esquizofrenias, consumo problemático de alcohol y drogas, trastornos orgánicos y trastornos de conducta.

De acuerdo a estos criterios se eligió:

- Consultante 1, adolescente mujer de 15 años, 2 meses de edad, hospitalizada en el servicio de psiquiatría y salud mental, cursando $1 .^{\circ}$ año de educación secundaria en colegio municipal, vive con adulto responsable, diagnóstico depresivo grave según Clasificación Internacional de Enfermedades en su décima versión (CIE-10), derivada de Servicio de Atención Médico de Urgencia (SAMU) por intento de suicidio con 40 fármacos y cortes en brazos y piernas. Estuvo en tratamiento con psicólogo en Centro de Salud Familiar (CESFAM) en los últimos seis meses.

- Consultante 2, adolescente mujer de 15 años, 6 meses de edad, hospitalizada en el servicio de psiquiatría y salud mental, cursando $2{ }^{\circ}$ año de educación secundaria en colegio municipal, vive con adulto responsable, con diagnóstico de depresión grave según CIE-10, derivada de la urgencia de un hospital público por intento de suicidio con 58 fármacos y cortes en brazos y piernas. Estuvo en tratamiento con psicólogo en Centro Comunitario de Salud Mental (COSAM) en los últimos cinco meses.

- Psicoterapeuta 1, psicólogo clínico del servicio de psiquiatría y salud mental de la infancia y de la adolescencia, 
con 10 años de antigüedad, acreditado por la CONAPC en el enfoque Construccionismo Social y 13 años de experiencia de atención a adolescentes.

- Psicoterapeuta 2, psicóloga clínica del servicio de psiquiatría y salud mental de la infancia y de la adolescencia, con 10 años de antigüedad, acreditada por la CONAPC en la línea Integrativa y con 8 años de experiencia de atención a adolescentes.

De esta forma, los casos de estudio fueron:
- 1. ${ }^{\circ}$ Caso de estudio que denominamos "Proceso 1 de psicoterapia", compuesto por Psicoterapeuta 1 y Consultante adolescente 1.

- 2. Caso de estudio que denominamos "Proceso 2 de psicoterapia”, compuesto por Psicoterapeuta 2 y Consultante adolescente 2 .

Los casos de estudio se resumen en la Tabla 1.

Tabla 1. Descripción de los casos de estudio (Proceso 1 - Proceso 2) de psicoterapia.

Proceso 1

\begin{tabular}{|c|c|c|}
\hline Paciente & Mujer 15 años, 2 meses & Mujer 15 años, 6 meses. \\
\hline Psicoterapeuta & $\begin{array}{l}\text { Hombre, } 13 \text { años de experiencia con } \\
\text { adolescentes }\end{array}$ & $\begin{array}{l}\text { Mujer, } 8 \text { años de experiencia con adoles- } \\
\text { centes. }\end{array}$ \\
\hline Derivada de & $\begin{array}{l}\text { SAMU, Intoxicada } 40 \text { fármacos, cortes } \\
\text { en brazos. }\end{array}$ & $\begin{array}{l}\text { UCI, intoxicada } 58 \text { fármacos y cortes en } \\
\text { brazos y piernas. }\end{array}$ \\
\hline Diagnóstico & Depresión Grave (CIE-10). & Depresión Grave (CIE-10). \\
\hline Tratamientos Previos & En CESFAM con psicólogo & COSAM con psicólogo \\
\hline Tratamiento a recibir & $\begin{array}{l}\text { Psicoterapia durante la hospitalización. } \\
\text { Se firman consentimientos. }\end{array}$ & $\begin{array}{l}\text { Psicoterapia durante la hospitalización. Se } \\
\text { firman consentimientos. }\end{array}$ \\
\hline $\begin{array}{l}\text { Total sesiones de psicoterapia } \\
\text { filmada }\end{array}$ & 14 sesiones. & 14 sesiones. \\
\hline $\begin{array}{l}\text { Sesiones de psicoterapia } \\
\text { analizada. }\end{array}$ & $1^{\circ}, 3^{\circ}, 6^{\circ}, 9^{\circ}, 12^{\circ} \mathrm{y} 14^{\circ}$ & $1^{\circ}, 3^{\circ}, 6^{\circ}, 9^{\circ}, 12^{\circ}$ y $14^{\circ}$. \\
\hline $\begin{array}{l}\text { Sesiones de psicoterapia } \\
\text { semanales. }\end{array}$ & $\begin{array}{l}2 \text { sesiones por semana, } 1 \text { de ella anali- } \\
\text { zada. }\end{array}$ & 2 sesiones por semana, 1 de ella analizada. \\
\hline Alta & $8^{\circ}$ semana. & $8^{\circ}$ semana. \\
\hline $\begin{array}{l}\text { Percepción de cambios de } \\
\text { consultantes }\end{array}$ & $\begin{array}{l}\text { Se puede controlar, piensa y reflexiona } \\
\text { antes de actuar, se proyecta. }\end{array}$ & $\begin{array}{l}\text { Es capaz de pensar y se concentra en buscar } \\
\text { soluciones, se controla, es más tolerante. }\end{array}$ \\
\hline
\end{tabular}

Fuente: Elaboración propia.

\section{Procedimientos}

\section{UNIDAD DE ANÁLISIS: ESTRUCTURA DE INTERCAMBIOS TERAPÉUTICOS}

Como método de descripción se utilizaron las categorías de análisis de la conversación (Kerbrat-Orecchioni, 1998). Para describir las conversaciones se utilizó como unidad de análisis el Intercambio, que es la unidad conversacional más pequeña que vincula unidades dialogales e interactivas del lenguaje — fases y bloques semánticos — con unidades monologales o contribución individual del discurso de cada interlocutor - intervenciones y actos de habla-.
Un estudio de psicoterapia con adolescentes de Barriga (2017), reconoció una "Estructura de Intercambios Terapéuticos" en la conversación psicoterapéutica, validadas y graficada en 6 tipos de Intercambios (Tabla $\mathrm{N}^{\circ} 2$ ), que agrupan diálogos producidos en las sesiones de psicoterapia y en la cual se centra este artículo. Las sesiones se segmentaron en fases o secuencias de tiempo denominadas inicio, desarrollo y cierre, en las cuales se analizaron los intercambios. 
Tabla 2. Estructura de Intercambios Terapéuticos.

\begin{tabular}{|c|c|c|}
\hline & Intercambios & Definición \\
\hline 1 & Adherencia & 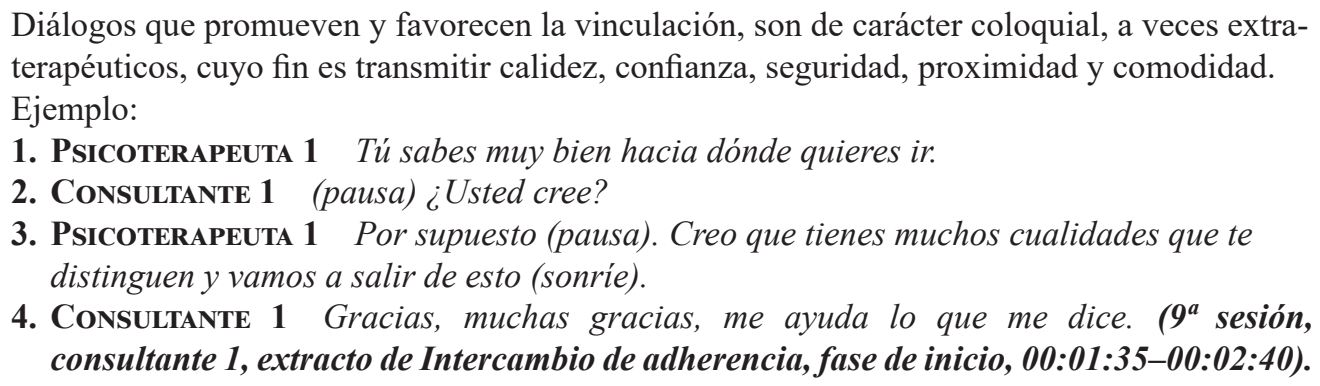 \\
\hline 2 & $\begin{array}{l}\text { Exploración del } \\
\text { síntoma }\end{array}$ & 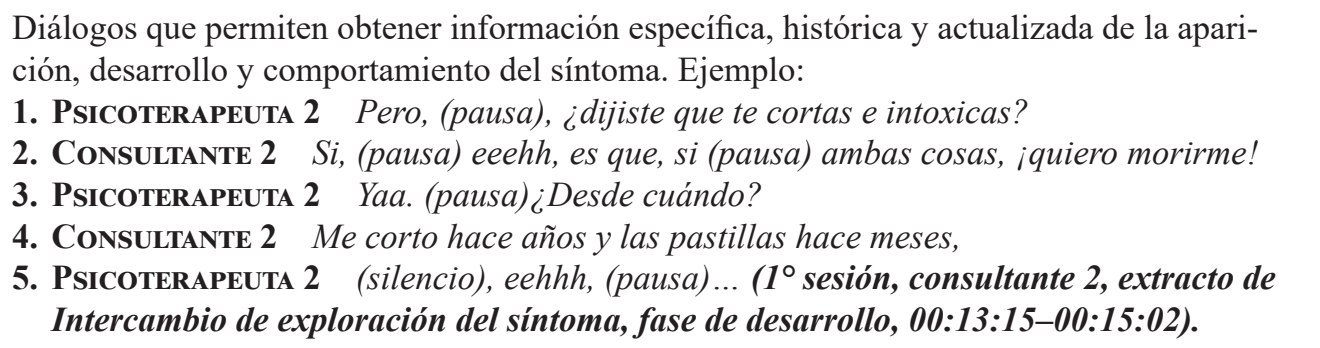 \\
\hline 3 & Relato subjetivo & 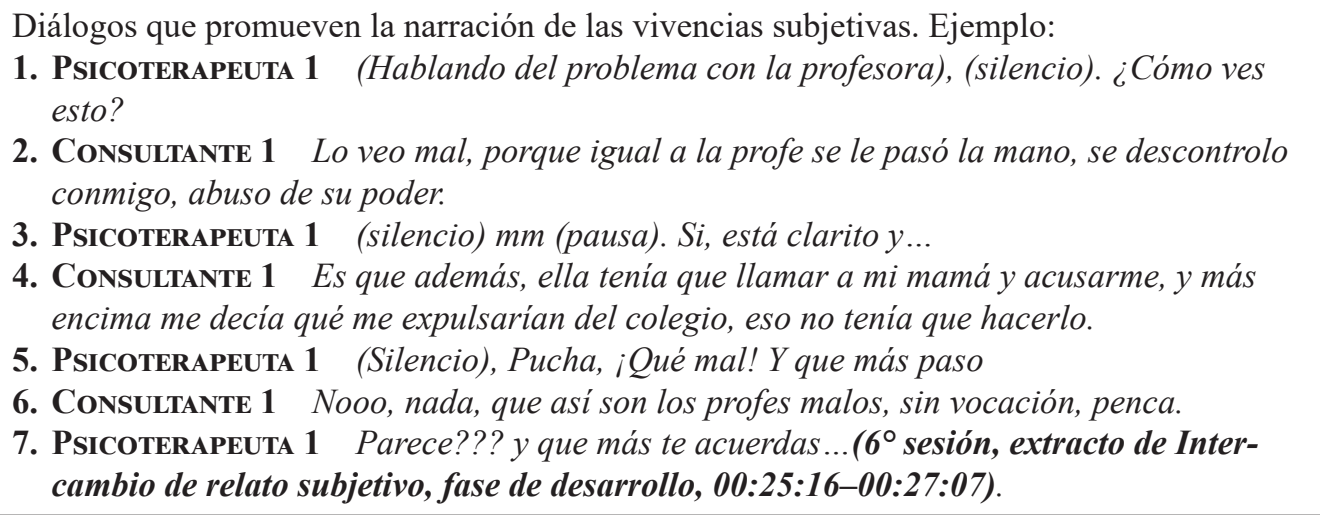 \\
\hline 4 & $\begin{array}{l}\text { Exploración del } \\
\text { contexto. }\end{array}$ & 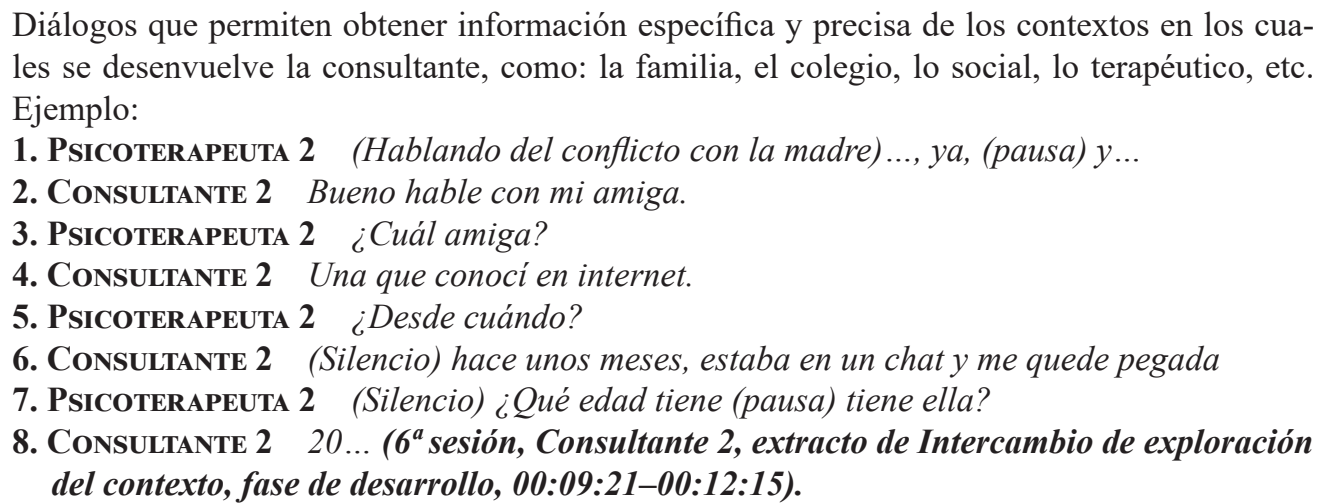 \\
\hline
\end{tabular}




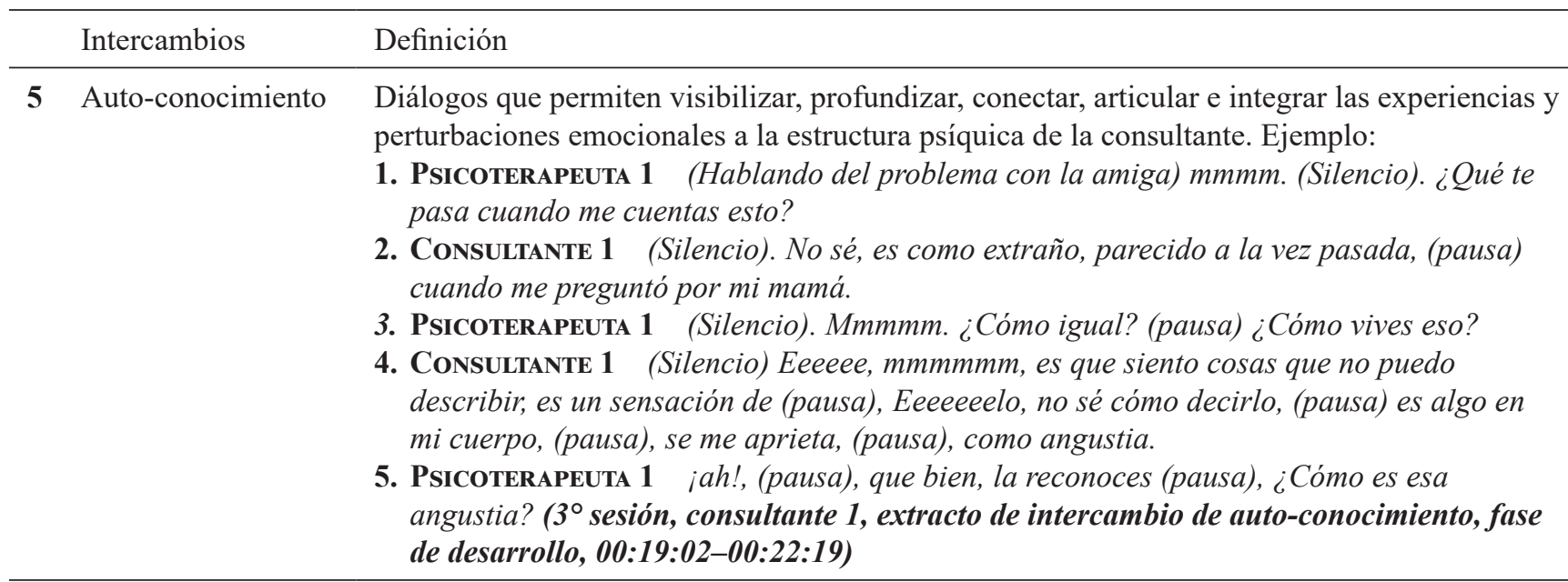

6 Explicación del problema del otro
Diálogos que permiten acuerdos y consenso sobre distintos estados internos de la consultante, tales como: malestar, sufrimiento, confusión, tranquilidad, entre otros. Ejemplo:

1. Psicoterapeuta 2 (consultante hablando de la consultante). Me da la impresión que cuando intentas resolver algo que no es tuyo te confundes.

2. Consultante 2 (Silencio). Eeeeh, (pausa) eeeeh.

3. Psicoterapeuta 2 (Silencio). Claro, porque hemos hablado y visto que de a poco intentas resolver tus problemas, (pausa) pero cuando interviene otro (pausa), tú como que te bloqueas.

4. Consultante 2 (Silencio). Es eso, me bloqueo, (pausa) no sé cómo reaccionar, (pausa) o reacciono mal, muy mal. $\left(12^{\circ}\right.$ sesión, consultante 2 , extracto de Intercambio de explicación del problema del otro, fase de desarrollo, 00:21:07-00:23:00).

Fuente: Elaboración propia.

\section{PROCESAMIENTO DE LA INFORMACIÓN}

Del Proceso 1 y Proceso 2 se analizaron las sesiones $1^{a}$, $3^{\mathrm{a}}, 6^{\mathrm{a}}, 9^{\mathrm{a}}, 12^{\mathrm{a}}$ y $14^{\mathrm{a}}$, con dos cámaras en modalidad fija y sincronizada al momento de analizar. Como la interacción entre psicoterapeuta y consultante es altamente dinámica, se optó por un procedimiento de observación continuo de las categorías de la estructura de intercambio, realizando marcas de tiempo cada 5 segundos durante el transcurso de las sesiones de psicoterapia. Este procedimiento permitió analizar asociaciones, frecuencia, duración, proporción y diferencias de tiempo real ocupado en la producción de cada categoría de Intercambio reconocida en la segmentación de las sesiones, ya sea en el intra o entre procesos de psicoterapia. El proceso de codificación se realizó con el software Videograph (Rimmele, 2009). Para el análisis de la concordancia inter-observador se aplicó el índice de Kappa, obteniéndose 0.842 (bueno) para las categorías de intercambio y de 0.823 (bueno) para las fases que segmentó a las sesiones. En el análisis de la significatividad de las asociaciones se utilizaron herramientas estadísticas no paramétricas como el Chi-Cuadrado de Pearson $\left(X^{2}\right)$, que por su sensibilidad al tamaño muestral se complementó con el coeficiente V de Cramer, que arroja una medida de asociación acotada e independiente del tamaño muestral, con valores entre $0=$ ausencia absoluta de relación, a $1=$ relación perfecta. En ciencias sociales, valores por sobre 0.30 indican asociación significativa moderada (Manzano-Arrondo, 2017). Adicionalmente, se analizó la dependencia en los casos con significatividad estadística entre las categorías, observándose la relación entre las frecuencias observadas y las esperadas en cada una de las casillas de la tabla de contingencia, a través del residuo tipificado corregido y las diferencias de las variables tipificadas. En este sentido, el nivel de relación de las categorías se determinó en la medida que $\mathrm{Z}= \pm 1.96$. El análisis comparativo de las sesiones intra o entre procesos se complementó con el usó de la prueba de U de Mann-Whitney.

\section{PROCEDIMIENTO ÉTICO}

Los protocolos del estudio fueron aprobados y autorizados para su implementación por el comité de bioética de la institución hospitalaria. Se firmó: Consentimiento informado los cuidadores; Consentimiento informado los psicoterapeutas y Asentimiento informado las consultantes. 


\section{Resultados}

Se presenta la conversación psicoterapéutica organizada en una Estructura de Intercambios Terapéuticos, analizando los tiempos y los movimientos interdependientes en las diferentes sesiones del trabajo psicoterapéutico en contexto hospitalario. Las sesiones fueron segmentadas en tres grandes fases de tiempo, denominadas inicio, desarrollo y cierre.

El inicio de la sesión es un tiempo de tránsito, que principalmente se caracteriza por la preparación al trabajo terapéutico, los psicoterapeutas en este segmento de tiempo se presentan, informan el proceso y obtienen información básica del contexto. El desarrollo corresponde a la fase de tiempo donde los psicoterapeutas comienzan a desarrollar el trabajo terapéutico aplicando sus conocimientos y estrategias de intervención. En la fase de cierre los psicoterapeutas entregan señales que la sesión está terminando, destacan temas tratados y preparan la despedida. En este sentido, el inicio y el cierre se caracterizan por ser fases temporales preparatorias, ya sea al trabajo terapéutico o al término de la sesión; en cambio, el desarrollo es la fase del trabajo psicoterapéutico que conduce al cambio. Las categorías de la Estructura de Intercambios Terapéuticos descritas en fases de segmentos temporales varían en el transcurso de las sesiones de los procesos de psicoterapia (Tabla $\mathrm{N}^{\circ} 3$ ).

En las 12 sesiones de psicoterapias analizadas -6 sesiones del Proceso 1 y 6 sesiones del proceso 2 - se contabilizaron 5.396 unidades de intercambios en tiempo constante de 5 segundos, de estas 2.602 unidades corresponden a intercambios del Consultante 1 y 2.794 unidades del Consultante 2.

Existe relación estadísticamente significativa entre la estructura de intercambios terapéuticos con los segmentos de tiempo de las sesiones de los procesos de psicoterapia [Chi cuadrado $(4, \mathrm{~N}=5,396)=76,39 ; \mathrm{p}<0.001]$, con una fuerza de dependencia moderada ( $V$ de Cramer $=0.301)$. Se infiere que la estructura de intercambios es interdependiente con la segmentación de las sesiones de psicoterapia en contexto hospitalario. Al analizar la distribución del tiempo de la estructura de intercambio entre los segmentos inicio, desarrollo y cierre, se encuentra que son estadísticamente diferentes (U de Mann-Whitney=317,733,55.141; $\mathrm{p}<0.001$ ). Es decir, los tiempos ocupados en los diferentes segmentos de las sesiones de ambos procesos, son sensibles a la estructura de intercambios terapéuticos.

Se observan diferencias significativas en la ocupación del tiempo de intercambios entre el proceso $1 \mathrm{y}$ el proceso 2 de psicoterapia $(Z=2.56 ; \mathrm{p}<0.05)$.

Tabla 3. Tiempos totales de la Estructura de Intercambios Terapéuticos al segmentar las sesiones en inicio, desarrollo y cierre, de los procesos 1 y 2 de psicoterapia (porcentajes).

\begin{tabular}{|c|c|c|c|c|c|c|c|c|}
\hline \multirow{2}{*}{$\begin{array}{l}\text { Estructura de } \\
\text { intercambio }\end{array}$} & \multicolumn{3}{|c|}{ Proceso 1} & \multirow{2}{*}{$\begin{array}{c}\text { Total } \\
1\end{array}$} & \multicolumn{3}{|c|}{ Proceso 2} & \multirow{2}{*}{$\begin{array}{c}\text { Total } \\
2\end{array}$} \\
\hline & INICIO & DESARROLLO & CIERRE & & INICIO & DESARROLLO & CIERRE & \\
\hline 1. Adherencia & $79.5[5.6]$ & $8.4[3.7]$ & $64.2[2.8]$ & 18.3 & $70.4[-5.6]$ & $5.3[-3.7]$ & $54.3[-2.8]$ & 12.2 \\
\hline $\begin{array}{l}\text { 2. Exploración } \\
\text { del síntoma }\end{array}$ & $0.5[-0.7]$ & $9.8[-2.1]$ & $8.1[-0.9]$ & 8.5 & $0.2[0.7]$ & $18.4[2.1]$ & $16.3[0.9]$ & 16.4 \\
\hline $\begin{array}{l}\text { 3. Relato } \\
\text { subjetivo }\end{array}$ & $1.2[-1.2]$ & $41.1[-7.2]$ & $9.4[-1.9]$ & 34.4 & $2.2[1.2]$ & $37.0[7.2]$ & $12.2[1.9]$ & 30.2 \\
\hline $\begin{array}{l}\text { 4. Exploración } \\
\text { del contexto }\end{array}$ & $18.4[3.3]$ & $11.1[1.6]$ & $6.2[-1.1]$ & 11.7 & $24.8[-3.3]$ & $19.4[-1.6]$ & $6.4[1.1]$ & 17.9 \\
\hline $\begin{array}{l}\text { 5. Auto- } \\
\text { conocimiento }\end{array}$ & $0.2[0.6]$ & $19.2[6.4]$ & $1.2[0.6]$ & 15.8 & $0.6[-0.6]$ & $15.1[-6.4]$ & $0.3[-0.6]$ & 11.6 \\
\hline $\begin{array}{l}\text { 6. Explicación } \\
\text { del problema del } \\
\text { otro }\end{array}$ & $0.2[-1.7]$ & $10.4[3.1]$ & $11.9[2.0]$ & 11.2 & $1.8[1.7]$ & $4.8[-3.1]$ & $10.1[-2.0]$ & 11.7 \\
\hline Total \% & 100 & 100 & 100 & 100 & 100 & 100 & 100 & 100 \\
\hline $\mathrm{T}(\mathrm{N})$ & 313 & 2,132 & 157 & 2,602 & 251 & 2,121 & 419 & 2,794 \\
\hline
\end{tabular}

[ ] $=$ Residuos tipificados corregidos. 
El análisis de los residuos tipificados corregidos de las casillas de la estructura de intercambios terapéuticos (Tabla $\mathrm{N}^{0} 3$ ), indican que los intercambios de exploración del síntoma, relato subjetivo, exploración del contexto y auto-conocimiento son independientes de los segmentos inicio y cierre de la sesión en ambos procesos. El análisis de los porcentajes de las casillas muestra que el inicio y cierre de las sesiones concentran la mayor cantidad de tiempo en el intercambio de adherencia, mientras que el desarrollo de las sesiones invierte mayor cantidad de tiempo en el intercambio de relato subjetivo y auto-conocimiento

Existe relación estadísticamente significativa entre los procesos de psicoterapia y la estructura de intercambios terapéuticos [Chi cuadrado $(5, \mathrm{~N}=5,396)=45,39$; $\mathrm{p}<0.001$ ], con una fuerza de dependencia moderada ( $\mathrm{V}$ de Cramer $=0.333$ ). Se infiere que la estructura de inter- cambios terapéuticos es interdependiente a los procesos de psicoterapia en contexto hospitalario.

Cuando se compara la distribución de los tiempos de las categorías de la estructura de intercambios durante el proceso de psicoterapia (Tabla $\mathrm{N}^{\circ} 4$ ), se encuentran que son estadísticamente diferentes (U de Mann-Whitney=24,555,667.544; $\mathrm{p}<0.001$ ). Es decir, la forma que los psicoterapeutas construyen y gestionan la psicoterapia es diferente en el transcurso del proceso e incide en el tiempo y en la organización del movimiento de las categorías de la estructura de intercambios terapéuticos durante las sesiones. En coherencia con lo señalado, se encuentran diferencias estadísticamente significativas en la distribución del tiempo ocupado en la producción de intercambios entre las sesiones de los procesos de psicoterapia (U de Mann-Whitney=25,478,125.557; $\mathrm{p}<0.005)$.

Tabla 4. Comparación U de Mann-Whitney de la distribución de los tiempos de las categorías de la estructura de intercambios terapéuticos en el proceso 1 y 2 de psicoterapia.

\begin{tabular}{lcccc}
\hline Procesos de Psicoterapia & $\mathrm{N}$ & $\begin{array}{c}\text { Rango } \\
\text { promedio }\end{array}$ & U de Mann-Whitney & $\begin{array}{c}\text { Sig. Asintótica } \\
\text { (bilateral) }\end{array}$ \\
\hline Proceso 1 & 2,602 & 1,852 & $24,555,667.554$ & 0.001 \\
\hline Proceso 2 & 2,794 & 2,129 & & \\
\hline Total $(\mathrm{N})$ & 5,396 & & & \\
\hline
\end{tabular}

Fuente: Elaboración propia.

Existe relación estadísticamente significativa entre la estructura de intercambios terapéuticos y la ocupación de tiempo de la $1^{\mathrm{a}}$ y $5^{\mathrm{a}}$ sesión del proceso 1 y 2 de psicoterapia [Chi cuadrado $(5, \mathrm{~N}=1,021)=284,214 ; \mathrm{p}<0.001 ; \mathrm{V}$ de Cramer $=0.332]$ y entre la $9^{\mathrm{a}}$ y $14^{\mathrm{a}}$ sesión del proceso 1 y 2 de psicoterapia [Chi cuadrado $(3, \mathrm{~N}=695)=211,211$; $\mathrm{p}<0.001$; V de Cramer $=0.301]$. Al analizar comparativamente la $1^{\mathrm{a}}$ y $5^{\mathrm{a}}$ sesión, y la $9^{\mathrm{a}}$ y $14^{\mathrm{a}}$ sesión, se observan diferencias significativas en los tiempos de las categorías de la estructura de intercambios en sesiones al interior de un mismo proceso $(Z=3.18 ; \mathrm{p}<0.01)$ y en sesiones entre los procesos $(Z=2.87 ; \mathrm{p}<0.04)$, lo que indica que la producción de intercambios es distinta entre sesiones de un mismo proceso, y también entre las sesiones de un proceso comparado con el otro.

El estudio analiza los tiempos de la estructura de intercambios de las sesiones $1^{\mathrm{a}}$ y $5^{\mathrm{a}}$ sesión (Tabla $\mathrm{N}^{\circ} 5$ ) y de la $9^{\mathrm{a}}$ y $14^{\mathrm{a}}$ sesión $\left(\right.$ Tabla $\left.\mathrm{N}^{\circ} 6\right)$, separados por procesos de psicoterapia. 
Tabla 5. Tiempos de la Estructura de Intercambios Terapéuticos de la $1^{\circ}$ y $5^{\circ}$ sesión por proceso de psicoterapia (porcentajes)

\begin{tabular}{|c|c|c|c|c|c|c|}
\hline \multirow{2}{*}{ Estructura de intercambio } & \multicolumn{2}{|c|}{ Proceso 1} & \multirow{2}{*}{$\begin{array}{c}\text { Total } \\
1\end{array}$} & \multicolumn{2}{|c|}{ Proceso 2} & \multirow{2}{*}{ Total 2} \\
\hline & $1^{\circ}$ Sesión & $5^{\circ}$ Sesión & & $1^{\circ}$ Sesión & $5^{\circ}$ Sesión & \\
\hline 1. Adherencia & $19.1[3.9]$ & $13.6[-3.9]$ & 17.4 & $15.7[3.3]$ & $11.3[-3.3]$ & 14.0 \\
\hline 2. Exploración del síntoma & $19.5[1.4]$ & $5.6[-1.4]$ & 14.2 & $24.5[1.8]$ & $6.4[-1.8]$ & 17.6 \\
\hline 3. Relato subjetivo & $35.9[-3.1]$ & $22.3[3.1]$ & 31.4 & $31.2[5.2]$ & $29.1[-5.2]$ & 30.4 \\
\hline 4. Exploración del contexto & $23.3[-1.9]$ & $4.8[1.9]$ & 16.1 & $27.3[2.7]$ & $8.1[-2.7]$ & 19.9 \\
\hline $\begin{array}{l}\text { 5. Auto- } \\
\text { conocimiento }\end{array}$ & $0.5[-6.4]$ & $29.2[6.4]$ & 13.1 & $0.1[-4.4]$ & $22.6[4.4]$ & 8.8 \\
\hline 6. Explicación del problema del otro & $1.7[-4.2]$ & $24.5[4.2]$ & 7.8 & $1.2[3.2]$ & $22.5[-3.2]$ & 9.3 \\
\hline Total \% & 100 & 100 & 100 & 100 & 100 & 100 \\
\hline $\mathrm{T}(\mathrm{N})$ & 278 & 204 & 462 & 345 & 214 & 559 \\
\hline
\end{tabular}

[ ] $=$ Residuos tipificados corregidos.

Tabla 6. Tiempos de la Estructura de Intercambios de la $9^{\circ}$ y $14^{\circ}$ sesión por proceso de psicoterapia (porcentajes).

\begin{tabular}{|c|c|c|c|c|c|c|}
\hline \multirow{2}{*}{ Estructura de intercambio } & \multicolumn{2}{|c|}{ Proceso 1} & \multirow{2}{*}{$\begin{array}{c}\text { Total } \\
1\end{array}$} & \multicolumn{2}{|c|}{ Proceso 2} & \multirow{2}{*}{ Total 2} \\
\hline & $9^{\circ}$ Sesión & $14^{\circ}$ Sesión & & $9^{\circ}$ Sesión & $14^{\circ}$ Sesión & \\
\hline 1. Adherencia & $12.3[3.1]$ & $19.6[-3.1]$ & 16.5 & $10.1[2.5]$ & $10.3[-2.5]$ & 10.2 \\
\hline 2. Exploración del síntoma & $1.5[-0.8]$ & $4.6[0.8]$ & 3.2 & $6.5[-1.9]$ & $7.4[1.9]$ & 6.9 \\
\hline 3. Relato subjetivo & $28.9[4.1]$ & $30.2[-4.1]$ & 29.6 & $32.9[5.4]$ & $29.3[-5.4]$ & 30.9 \\
\hline 4. Exploración del contexto & $3.3[-1.1]$ & $6.8[1.1]$ & 5.3 & $5.3[-1.8]$ & $8.4[1.8]$ & 7.0 \\
\hline $\begin{array}{l}\text { 5. Auto- } \\
\text { conocimiento }\end{array}$ & $38.8[4.9]$ & $18.6[-4.9]$ & 24.8 & $29.4[3.4]$ & $15.2[-3.4]$ & 21.4 \\
\hline 6. Explicación del problema del otro & $21.2[2.3]$ & $20.2[-2.3]$ & 20.6 & $15.8[-2.2]$ & $29.4[2.2]$ & 23.6 \\
\hline Total \% & 100 & 100 & 100 & 100 & 100 & 100 \\
\hline $\mathrm{T}(\mathrm{N})$ & 147 & 191 & 338 & 156 & 201 & 357 \\
\hline
\end{tabular}

[ ] $=$ Residuos tipificados corregidos. 
El análisis comparativo de los residuos tipificados corregidos de las casillas de la estructura de intercambios de la $1^{\circ}$ y $5^{\circ}$ sesión separados por procesos (Tabla $\mathrm{N}^{\circ} 5$ ), indican que en el proceso 1 las categorías de intercambios exploración del síntoma y exploración del contexto son independiente de los procesos de psicoterapia, en tanto, en el proceso 2 sólo el intercambio exploración del síntoma es independiente. Se advierte que, en la $1^{\circ}$ sesión de ambos procesos, los psicoterapeutas invierten más tiempo en el intercambio de adherencia y menos tiempo en el intercambio de auto-conocimiento, y que en la $5^{\circ}$ sesión de ambos procesos se invierte más tiempo en el intercambio de auto-conocimiento. Se observa en el proceso 1 , que la $5^{\circ}$ sesión comparada con la $1^{\circ}$, se gasta más tiempo en intercambios de auto-conocimiento y explicación del problema, mientras que en el proceso 2 , la $5^{\circ}$ sesión invierte menos tiempo en el intercambio de explicación del contexto al compararla con la $1^{\circ}$ sesión. Por otra parte, el análisis de los porcentajes de las casillas indica que el intercambio de relato subjetivo concentra la mayor cantidad de tiempo en la estructura de intercambios terapéuticos en ambas sesiones $\left(1^{\circ}\right.$ y $\left.5^{\circ}\right)$ y en ambos procesos de psicoterapia ( 1 y 2$)$; y que el intercambio exploración del síntoma y exploración del contexto concentran más tiempo en la $1^{\circ}$ sesión y son independiente del proceso.

El análisis comparativo de los residuos tipificados corregidos de las casillas de la estructura de intercambios de la $9^{\circ}$ y $14^{\circ}$ sesión (Tabla Nº), indican que las categorías de intercambio exploración del síntoma y exploración del contexto son independiente en ambos procesos de psicoterapia. Además, el intercambio de auto conocimiento ocupa menos tiempo en la última sesión en ambos procesos de psicoterapia. Llama la atención que en la última sesión del proceso 2 se ocupa más tiempo en el intercambio de explicación del problema del otro.

El análisis de los porcentajes de las casillas indica que el intercambio de adherencia y de relato subjetivo presenta una producción estable durante las sesiones analizadas en ambos procesos.

\section{Discusión y Conclusiones}

El artículo se focaliza en las características del uso del tiempo de las distintas categorías de intercambios, segmentando las sesiones en inicio, desarrollo y cierre para una mayor comprensión de los movimientos de la Estructura de Intercambios Terapéuticos, analizada en la conversación de psicoterapias completas de adolescentes depresivos con intento de suicidio en contexto de salud pública hospitalaria. Los intercambios en términos de Kerbrat-Orecchioni (1998) constituyen la unidad mínima dialogal que vincu- la a interlocutores mutuamente orientados, observándose que esta unidad configurada en una estructura presenta una ocupación de tiempos diferentes en el transcurso de cada proceso de psicoterapia, que incide y produce un dinámico movimiento en la producción de intercambios que caracteriza la interacción interdependiente, cooperativa y coordinada de psicoterapeutas con consultantes-adolescentes. En este sentido, es posible pensar que los intercambios se gestionan de acuerdo a la utilidad que ofrecen en el trabajo psicoterapéutico, es decir, las sesiones funcionan con una producción de intercambios cuyo fin último es buscar la construcción conjunta de significados para aproximarse al cambio terapéutico en contexto hospitalario, en consecuencia, la ocupación de intercambios es sensible y se ajusta al trabajo y objetivos terapéuticos del psicoterapeuta que van surgiendo en la interacción.

Al haber más tiempo para el intercambio de adherencia al inicio - cierre de cada sesión y de manera estable en ambos procesos psicoterapéuticos, permite ver que generar comodidad tiene que estar presente en todas las sesiones, que es un trabajo permanente del psicoterapeuta, y que el intercambio de adherencia posee una doble función, ya que no solo se perfila como generador de alianza terapéutica al promover diálogos de cordialidad, confianza y comodidad, asegurando el bienestar personal del consultante, sino también se constituye en un mecanismo básico de acción intersubjetiva que garantiza, por un lado, la mutua sintonización entre interlocutores que permite al inicio de la sesión bajar las posibles resistencias que el consultante adolescente puede traer a psicoterapia, y por otro, reparar al cierre del encuentro cualquier aspereza que se haya producido en la sesión, volviendo a re-sintonizar con el adolescente, y así re-conectarlo al continuo del proceso terapéutico que volverá a tomar vida en una próxima sesión. Es probable que el intercambio de adherencia constituya un soporte clave al inicio y cierre de cada encuentro conversacional, y además una estrategia psicoterapéutica imprescindible que sienta las bases de la edificación del cambio. Esto es concordante con teorías de autores como Rogers (1969), Orlinsky \& Howard (1986), quienes documentan que el éxito de la psicoterapia pasa por el desarrollo de una buena alianza y adherencia terapéutica.

Que el intercambio de auto-conocimiento presente una baja producción en la $1^{\circ}$ y $14^{\circ}$ sesión y que sea independiente de los procesos en las fases de inicio-cierre, pero con una alta ocupación en la fase de desarrollo de las sesiones, nos permite afirmar que es un intercambio exclusivo de trabajo psicoterapéutico, ya que para producir diálogos que implican exploración, identificación y expresión emocional, primero es necesario una adecuada adherencia del consultante adolescente, que puede explicar su ausencia en la $1^{\circ}$ sesión, y segundo, necesita ocupar mayor cantidad de 
tiempo para acceder, movilizar y articular diferentes contenidos emocionales del adolescente, que puede explicar su presencia en la fase de desarrollo. Esto es concordante con la postura de Guidano (1999), quien plantea que el psicoterapeuta debe focalizarse y trabajar en y con el mundo emocional del otro para provocar el cambio, de forma que lo emocional se integre a su estructura psíquica; y también con las contribuciones de Arciero (2009), quien argumenta que las perturbaciones emocionales necesitan tiempo para trabajarlas y articularlas. Desde esta perspectiva, pensamos que la ocupación del intercambio de auto-conocimiento es estratégico, es decir, se debe saber cómo y cuándo ocuparlo, ya que estos diálogos que se promueven en la intersubjetividad del encuentro, tienen que entrar en acción sólo al existir adherencia y el tiempo suficiente para abrir y cerrar este intercambio. Se desprende que su utilización es con cautela y que al realizar un trabajo focalizado en el mundo emocional del consultante, constituye un tipo de intercambio de trabajo emocional.

Llama la atención que el intercambio de relato subjetivo presenta la mayor ocupación de tiempo y una producción estable durante los procesos de psicoterapia. Se infiere que los psicoterapeutas buscan generar un dialogo detallado, extendido y exhaustivo que permita recordar y describir, paulatina y progresivamente distintos contenidos de la historia del adolescente, de manera de obtener un conocimiento amplio, privilegiado, preciso, específico y saturado de su vivencia. En este sentido, pensamos que este intercambio opera para el psicoterapeuta como una columna transversal que provee, moviliza y conecta información, configurando una panorámica general del adolescente que orienta al psicoterapeuta y la psicoterapia. Esto es concordante con Peräkyla (2008), quien plantea que en la intersubjetividad del encuentro conversacional se construye la historia del paciente y la comprensión de las prácticas organizativas de la psicoterapia.

Que los intercambios de exploración del síntoma y exploración del contexto presenten más tiempo en la $1^{\circ}$ sesión y en general sean independiente del proceso, nos lleva a pensar que la producción de estos intercambios son más funcionales al inicio del proceso psicoterapéutico, ya que al dialogar sobre la naturaleza del síntoma y los elementos del contexto circundante, se accede al mundo más manifiesto, visible y reconocido por los adolescentes, y entendemos que este tipo de diálogos ayudara a bajar las resistencia del adolescente a conversar, y que en las siguientes sesiones su producción se reduce al mínimo para dar paso a otros intercambios. Conversar temas fácilmente reconocidos por los interlocutores, ha sido propuesto por Pomerantz \& Fehr (2000), al plantear que para entender la coordinación de acciones y las emisiones verbales de los interlocuto- res es necesario abordar temáticas con diferentes niveles de profundidad.

Pensamos que la diferencia encontrada en los tiempos de producción de las categorías de intercambios en las sesiones entre los procesos, era esperable, ya que los psicoterapeutas basados en sus propios modelos teóricos-prácticos de formación presentan formas distintas de establecer contacto con el consultante, de utilizar el lenguaje y de aplicar sus técnicas, pero esto no afecta el contenido que caracteriza a cada intercambio ni a los procedimientos establecidos por la institución de salud. No obstante, en futuros estudios es necesario profundizar la posible relación entre tiempos de producción de categorías de intercambios, orientaciones y género de los psicoterapeutas.

En conclusión, en los procesos psicoterapéuticos de adolescentes en contexto hospitalario, la estructura de intercambios terapéuticos presenta un dinámico movimiento posible de describir entre los procesos y entre las sesiones de cada proceso, siempre con la lógica de ajustarse a la gestión del trabajo psicoterapéutico, el que surge en la interacción de los interlocutores. Creemos que este conocimiento puede ayudar al clínico que trabaja con adolescentes, al considerar que:

- Existen intercambios que son más funcionales cuando se producen al inicio del proceso, como el intercambio de exploración del síntoma y exploración de contexto.

- Existe un intercambio que tiene que producirse durante todo el proceso, especialmente, en la fase de inicio y cierre de cada sesión, que es el intercambio de adherencia.

- Existe un intercambio que tiene que utilizarse con cautela, ya que trabaja a nivel emocional, que es el intercambio de auto-conocimiento.

En este sentido, el psicoterapeuta tiene que saber qué, cuándo y cómo utilizar la Estructura de Intercambios Terapéuticos.

No obstante, al ser un estudio exploratorio y descriptivo tiene que continuar desarrollándose y buscar mayor precisión de la "Estructura de Intercambios terapéuticos" descrita, e indagar en estructuras dialogales de psicólogos con distintas formaciones psicoterapéuticas y en diferentes consultantes en cuanto a lugares de atención, tipo de patología, rango de edad, grupos de migrantes, entre otros. 


\section{Referencias}

Almonte, C. \& Montt, M. (2012). Psicopatología infantil y de la adolescencia $\left(2^{\circ}\right.$ edición). Ed. Mediterraneo, Santiago.

Aristegui, R., Reyes, L., Tomicic, A., Vilches, O., Krause, M., De la Parra, G., Ben Dov, P., Dagnino, P., Echávarri, O. \& Valdés, N. (2004). Actos de habla en la conversación terapéutica. Terapia Psicológica, 22, 131-143.

Arciero, G. (2009). Selfhood, Identity and Personality Styles. New York: Wiley.

Barrientos, J. \& Cárdenas, M. (2013). Homofobia y Calidad de Vida de Gay y Lesbianas: Una Mirada Psicosocial. Psykhe, 22, 3-14.

Barriga, L. (2017). El Cambio en la Psicoterapia: Análisis de la Conversación en Psicoterapias con adolescentes mujeres hospitalizadas por intento de suicidio en un Hospital de le Región Metropolitana de Chile. (Tesis para optar al grado de Doctor en Psicología), Programa de Doctorado, Escuela de Psicología, Universidad de Santiago de Chile, 2017.

Baylis, P., Collins, D. \& Coleman, H. (2011). Child Alliance Process Theory: A Qualitative Study of a Child Centred Therapeutic Alliance. Child and Adolescent Social Work Journal, 28, 79-95.

Forman, S. (2004). Evidence-based psychotherapies for children and adolescents. Psychotherapy Research, 14, 488-490.

Gaete, J., Aristegui, R. \& Krause, M. (2017). Cuatro prácticas conversacionales para propiciar un cambio de foco terapéutico. Revista Argentina de Clínica Psicológica, 28, 220-231.

Guajardo, N., Ojeda, F., Achui, L. \& Larraguibel, M. (2015). Intervenciones Terapéuticas para la Conducta Suicida en Adolescentes. Revista chilena de psiquiatría y neurología de la infancia y adolescencia, 26, 145-155.

Guidano, V. (1999). Desarrollo de la Terapia Cognitiva Post-Racionalista. Santiago: Instituto de Terapia Cognitiva.

Hernández, M. (2000). Comer gente: un análisis desde la etnografía del habla. Revista de Filología y Lingüistica de la Universidad de Costa Rica, 26, 169-188.

Jiménez, A. \& Aceituno, R. (2013). El suicidio de Alejandra. Santiago de Chile: Centro de Investigación Periodística. Recuperado desde https:// ciperchile.cl/el-suicidio-de-alejandra/

Kerbrat-Orecchioni, C. (1998). Les inteactions verbales. Approche interactionnelle et structure desconversations. Tome I. Troisiéme Èdition. Armand Colin. Paris, France.

Kondratyuk, N. \& Peräkylä, A. (2011). Therapeutic work with the present moment: A comparative conversation analysis of existential and cognitive therapies. Psychotherapy Research, 21, 316-330.

Manzano-Arrondo, V. (2017). Chi cuadrado de Pearson para dos variables nominales. Recuperado desde http://asignatura.us.es/dadpsico/ apuntes/ChiCuadrado.pdf

Ministerio de Salud de Chile (2013). Programa Nacional de prevención del suicidio: orientaciones para su implementación. Departamento de salud mental, división de prevención y control de enfermedades, subsecretaria de salud pública.

Ministerio de Salud de Chile (2000). Plan Nacional de Salud Mental y Psiquiatría. Santiago: MINSAL, Sección salud mental.
Organización para la Cooperación y el Desarrollo Económicos (OCDE) (2013). Health at a Glance. OECD Indicators. Organización para la cooperación y el desarrollo económico.

Orlinsky, D. \& Howard, K. (1986). Process and outcome in psychotherapy. En: Garfield y Bergin, Handbook of psychotherapy and behavior change (3ra. Ed.). New York: Wiley.

Peräkyla, A. (2004). Two traditions of interaction. Department of Sociology, University of Helsinki, Finland British. Journal of Social Psychology, 43, 1-20.

Peräkylä, A. (2008). Conversation analysis and psychoanalysis: interpretation, affect, and intersubjectivity. In EBAPE (ed.) Conversation analysis and psychotherapy (pp. 100-119). Cambridge: Cambridge University Press.

Pomerantz, A. \& Fehr, B. (2000). Análisis de la conversación: enfoque del estudio de la acción social como prácticas de producción de sentido. En DIJK, Teun A. Van (ed). El discurso como interacción social (pp. 101-139). Barcelona: Gedisa.

Rimmele, R. (2016). The program for coding of videos. Recuperado desde www.dervideograph.de

Rogers, R. (1969). The necessary and sufficient conditions of therapeutic personality change. Journal of Consulting Psychology, 21, 95-103.

Sack, H., Schegloff, E. \& Jefferson, G. (1974). A simple's systematics for the organization of turn-taking for conversation. Language, 50, 696-735.

Sallés, P (2011). Elección de un psicólogo. Recuperado desde https://www. guioteca.com/psicologia-y-tendencias/como-elegir-a-su-psicologo/

Stake, R. (2005). Investigación con estudio de casos. Madrid: Morata.

Vargas, B., Gumucio, F. \& González, C. (2015). Efectividad en intervenciones terapéuticas con niños y adolescentes: Factores asociados a la Persona del Terapeuta y la Alianza Terapéutica. Revista chilena de psiquiatría y neurología de la infancia y adolescencia, 26, 7-10.

Villalta, M. (2009). Análisis de la Conversación. Una propuesta para el estudio de interacción didáctica en sala de clases. Estudios pedagógicos, 35, 221-238.

Vicente, B., Saldivia, S., Rioseco, P., De la Barra, F., Valdivia, M., Melipillan R., Zúñiga, M., Escobar, B. \& Pihán, R. (2010). Epidemiologia de trastornos mentales infanto-juveniles en la Provincia de Cautín. Revista Médica de Chile, 138, 965-973.

Vicente, B., Saldivia, S. \& Pihán, R. (2016). Prevalencias y brechas hoy: salud mental mañana. Acta bioethica, 22(1), 51-61 